\title{
Relevancia del patrón de persistencia de Hurst en la gestión de portafolios de renta variable
}

\author{
MARTínez PATIÑO, MANUEL ANDRÉS \\ Universidad de la Salle (Bogotá, Colombia) \\ Correo electrónico: manuelmartinez@unisalle.edu.co \\ ARIZA GARZÓN, MILLER JANNY \\ Universidad Piloto de Colombia (Bogotá, Colombia) \\ Correo electrónico: miller-ariza@unipiloto.edu.co \\ CADENA LOZANO, JAVIER BERNARDO \\ Colegio de Estudios Superiores de Administración (Bogotá, Colombia) \\ Correo electrónico: javier.cadena@cesa.edu.co
}

\begin{abstract}
RESUMEN
En este artículo se analiza el comportamiento de los retornos de algunos activos del MILA (Mercado Integrado Latinoamericano), con el objetivo de buscar evidencia de persistencia y evaluar el impacto de su presencia en la toma de decisiones de carteras de inversión. Se usó la metodología del rango reescalado en la estimación del coeficiente de Hurst como una medida de la persistencia y se comprueban los resultados con el ajuste de Anis y Lloyd y la estimación de Higuchi. Se incluye un proceso inferencial sobre el coeficiente de Hurst para cada uno de los activos analizados. Comparamos el desempeño de optimizar portafolios incluyendo estimaciones de persistencia y los resultados de su inferencia con portafolios gestionados bajo independencia. Se observa una mejor relación de riesgo-rendimiento al incluir el patrón de persistencia, solo cuando la inferencia así lo evidencia.
\end{abstract}

Palabras clave: persistencia, dependencia a largo plazo, rango reescalado, optimización de portafolios, coeficiente de Hurst.

Clasificación JEL: G17; G11; G15.

MSC2010: 60G22; 65K10. 


\title{
Relevance of Hurst's pattern in equity portfolio management
}

\begin{abstract}
In this article, the behavior of the returns of some assets of MILA is analyzed, with the objective of looking for evidence of persistence and evaluating the impact of their presence in the decision making of investment portfolios. The methodology of the rescaled range is used in the estimation of the Hurst coefficient as a measure of persistence and the results are verified with the adjustment of Anis and Lloyd and the estimation of Higuchi. An inferential process is added to the Hurst coefficient for each of the assets analyzed. The performance of portfolio optimization including estimates of persistence and the results of its inference were compared with independently optimized portfolios. A better risk-return relationship is observed by including the pattern of persistence, only when the inference is supported by evidence.
\end{abstract}

Keywords: persistence, long term dependency, rescaled rank, portfolio optimization, Hurst estimation.

JEL classification: G17; G11; G15.

MSC2010: 60G22; 65K 10 .

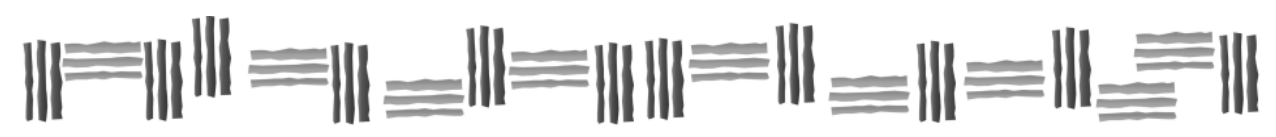




\section{Introducción.}

Esta investigación tiene como objetivo analizar la persistencia de las series temporales de algunos activos financieros del mercado MILA y a su vez reconocer su efecto en la optimización de portafolios. Esta medición, aplicada inicialmente por Mandelbrot y Van Ness (1968) en desarrollo de los procesos fraccionales, consiste en el estudio de la geometría irregular. El nombre fractal o fraccional lo determinan Mandelbrot y Wallis (1969) en sus primeras publicaciones y más adelante lo profundiza con los estudios de series financieras y su relación con los procesos de memoria de largo plazo. El concepto de persistencia se ha desarrollado de manera simultánea con las teorías basadas en los supuestos de independencia de las series financieras.

El método de estimación para determinar si una serie contiene un patrón de memoria de largo plazo fue desarrollado por Harold Edwin Hurst, como se presenta en Mandelbrot y Hudson (2005), quien se dedica a estudiar los movimientos del cauce del río Nilo en Egipto. En su estudio concluye que, con ayuda de la descomposición de la varianza de la serie en bloques o por segmentos, se pueden observar similitudes entre varios períodos y establecer la influencia de los datos históricos sobre los recientes. Intuitivamente se sabe que los egipcios tenían métodos para prever cambios en el cauce del río Nilo y así establecer los períodos de cosecha, pero además de eso, encuentra que el cauce podía estar asociado con recorridos del río que se dieron mucho tiempo atrás y que marcan la tendencia del nuevo cauce del río.

Este trabajo se desarrolla de la siguiente manera. Inicialmente se estudia desde la teoría el patrón de persistencia a partir de los movimientos fraccionales brownianos ( $\mathrm{fBm}$ ) que, a diferencia de los movimientos brownianos, no asumen independencia de las observaciones. Se presenta el proceso teórico para la obtención del parámetro de Hurst con base en la metodología de rango reescalado, se realiza también la descripción del ajuste de Anis y Lloyd (1976) y su proceso inferencial, que se complementa con la propuesta del q óptimo de Lo (1991) y los desarrollos de Teverovsky, Taqqu y Willinger (1999) y Willinger, Taqqu y Teverovsky (1999). Se describe también el proceso de estimación de Higuchi para comparar las estimaciones realizadas.

Finalmente, se presenta el proceso de optimización de portafolios incluyendo el patrón de persistencia sobre los desarrollos de Sharpe a través de la propiedad de autosimilitud y se evalúa el desempeño de incluir y no incluir el patrón de persistencia, reconociendo el proceso inferencial. El resto del documento se organiza como sigue. Una descripción teórica con los tópicos descritos, una sección de estado del arte que describe el uso del patrón de persistencia en estudios que analizan portafolios con diferentes activos financieros, una sección que describe los datos y la metodología propuesta, una sección de resultados y una última sección que resume las principales conclusiones y contribuciones derivadas de los hallazgos encontrados.

\section{Movimiento fraccional Browniano.}

Un movimiento fraccional browniano $(\mathrm{fBm})$ es un proceso estocástico que se divide principalmente en tres categorías según el exponente en el que se encuentre el parámetro de persistencia $H \in(0,1)$, o parámetro de Hurst. En particular, se considera un proceso gaussiano $B_{t}^{H}, t \geq 0$, con $E\left(B_{t}^{H}\right)=0$ con estructura de covarianza, como se expone en (1), de acuerdo con Hu y Øksendal (2003).

$$
E\left(B_{t}^{H} B_{s}^{H}\right)=\frac{1}{2}\left(s^{2 H}+t^{2 H}-|t-s|^{2 H}\right)
$$

Con $s \leq t$ y $s, t \geq 0$, tiempos definidos. Por simplicidad, $B_{0}^{H}=0$. Si el parámetro de Hurst $H$ toma un valor $H<\frac{1}{2}$ entonces la serie mostrará antipersistencia, en cambio si $H=\frac{1}{2}, B_{t}^{H}$ coincidirá con el movimiento Browniano y la varianza será igual a $t$. Por último, si el parámetro $H>\frac{1}{2}$ entonces 
la serie mostrará persistencia, memoria o dependencia de largo plazo. Si bien la varianza puede variar cuando el valor de $H$ cambia, el valor esperado sigue siendo igual a cero.

Por lo tanto, la varianza del diferencial $B_{t}^{H}-B_{s}^{H}$ se define como:

$$
E\left[\left(B_{t}^{H}-B_{s}^{H}\right)^{2}\right]=|t-s|^{2 H}
$$

Se puede ver en (2) que si $H=\frac{1}{2}$ entonces la $\operatorname{Var}\left(B_{t}^{H}-B_{s}^{H}\right)=t-s$.

Si $B_{t}^{H}$ tiene un valor inicial $B_{0}^{H}$ en $t=0$, entonces el cambio acumulado de $B_{t}^{H}$ desde $t=0$, de acuerdo con Mandelbrot y Wallis (1969), está dado por:

$$
B_{t}^{H}-B_{0}^{H}=\int_{-\infty}^{t}\left[(t-u)^{H-\frac{1}{2}}-N(u)(-u)^{H-\frac{1}{2}}\right] d B_{u}, 0<H<1
$$

donde $N(u)=1$, si $-\infty<u \leq 0$ y $N(u)=0$, para $u>0$. Si $H=\frac{1}{2}$, como ya se mencionó, se tiene el movimiento Browniano.

La suposición de independencia de los datos de una serie ayuda en el cálculo y solución de los procesos estocásticos, pues éstos se pueden resolver a través de métodos de probabilidad con ayuda de las propiedades de los procesos martingala, o resolviendo las ecuaciones parciales diferenciales directamente y la integral estocástica con ayuda de las integrales Ito. Sin embargo, cuando se considera un proceso fraccional existen problemas de convergencia y el valor esperado debe ser estimado. Para esto, con ayuda del cálculo Mallavian propuesto por Benth (2003), se propone una solución que, si bien no se puede generar a partir de una martingala, si con una cuasi-martingala, término nombrado por $\mathrm{Hu}$ y Øksendal (2003).

Por su parte, Nualart (2003) con base en una solución presentada por Cheridito (2001), con respecto a una semi martingala débil, describe el intervalo de un proceso fraccional browniano cuando $H>\frac{1}{2}$. Se busca con esto, una solución en donde el fBm sea una generalización del proceso browniano bajo ciertos parámetros a través de los que se pueda representar la covarianza cuando ésta muestra el patrón de persistencia.

\section{Incorporación de $\mathrm{H}$ en el cálculo del parámetro beta de los activos financieros para la optimización de portafolios.}

Los incrementos del movimiento fraccional browniano $B_{H}(t+s)-B_{H}(t)$ son variables aleatorias idénticamente distribuidas normales, con dependencia de largo plazo. Siguiendo a Mandelbrot y Van Ness (1968), el movimiento browniano fraccional es un proceso autosimil para todo $t$ y $s$, así:

$$
B_{H}(t+s)-B_{H}(t) \triangleq S^{H}\left[B_{H}(t+1)-B_{H}(t)\right]
$$

donde, $\triangleq$, representa idéntico en distribución. Derivado de esto:

$$
E\left[B_{H}(t+s)-B_{H}(t)\right]^{2}=S^{H} E\left[B_{H}(t+1)-B_{H}(t)\right]^{2}
$$

con:

$$
E\left[B_{H}(t+s)-B_{H}(t)\right]=S^{H} E\left[B_{H}(t+1)-B_{H}(t)\right]=0
$$

Y así, de (4), (5) y (6), se tiene: 


$$
\begin{aligned}
& \operatorname{Cov}\left\{\left[B_{H_{j}}(t+s)-B_{H_{j}}(t)\right],\left[B_{H_{0}}(t+s)-B_{H_{0}}(t)\right]\right\} \\
= & S^{H_{j}+H_{0}} \operatorname{Cov}\left\{\left[B_{H_{j}}(t+1)-B_{H_{j}}(t)\right],\left[B_{H_{0}}(t+1)-B_{H_{0}}(t)\right]\right\}
\end{aligned}
$$

Para generar los retornos por activo, Greene y Fielitz (1980) se basan en el modelo de mercado propuesto por Sharpe (1963), en donde se genera un portafolio a partir de los movimientos brownianos del índice de mercado y del activo y se incorpora la teoría de los modelos fraccionales, en particular, el patrón de persistencia. Los retornos de cada uno de los activos, en el modelo de Sharpe (1963) se trabajan con incrementos a partir de movimientos brownianos que asumen independencia y se definen como $B_{j}(t)=\beta B_{0}(t)+E_{j}(t)$ donde $\beta_{j}$ es una constante y $E_{j}(t)$ es un proceso estocástico estacionario conocido como movimiento browniano con parámetro $H=\frac{1}{2}$. En este caso, con ayuda de la solución de Nualart (2003) y Biagini, Campanino, Fuschini (2008) se trabaja de manera homóloga, pero asumiendo fBm para cada uno de los activos.

$$
B_{t}^{H}=\beta_{j} B_{0}^{H}(t)+E_{j}(t)
$$

Greene y Fielitz (1980) para incorporar la dependencia de largo plazo en la generación de retornos a partir de (3) presentan:

$$
\begin{aligned}
& B_{t}^{H_{0}}-B_{0}^{H_{0}}=\int_{t}^{\infty}\left[(t-s)^{H_{0}-\frac{1}{2}}-N(s)(-s)^{H_{0}-\frac{1}{2}}\right] d B_{s_{0}} \\
& B_{t}^{H_{j}}-B_{0}^{H_{j}}=\int_{t}^{\infty}\left[(t-s)^{H_{j}-\frac{1}{2}}-N(s)(-s)^{H_{j}-\frac{1}{2}}\right] d B_{s_{j}}
\end{aligned}
$$

donde $B_{t}^{H_{0}}$ hace referencia al mercado y $B_{t}^{H_{j}}$ al activo $j$. Una vez que se obtienen los rendimientos de cada uno de los activos, se calcula la covarianza, con el objetivo de estimar el riesgo de cada activo en un intervalo de tiempo $s$. Lo anterior soporta también la obtención de (7). Finalmente, para incorporar la tendencia, donde $d$ representa el diferencial del tiempo, en los incrementos del activo y del mercado, se tiene:

$$
\begin{aligned}
& R_{0}(t, d)=B^{H_{0}}(t+d)-B^{H_{0}}(t)+\alpha_{0} d, \\
& R_{j}(t, d)=B^{H_{j}}(t+d)-B^{H_{j}}(t)+\alpha_{j} d,
\end{aligned}
$$

Dado que estos incrementos presentan el mismo comportamiento de los ruidos fraccionales con media cero, después de corregir por la media, se puede obtener el estimador para el modelo que permite cuantificar el riesgo sistemático de un activo en función de los retornos del índice de mercado, a su vez, en función de $d$ y de las constantes estimadas de Hurst para cada variable, a partir de la regresión de $R_{j}(t, d)$ sobre $R_{0}(t, d)$, específicamente sobre los retornos continuos, para un tamaño de muestra $n$ :

$$
\hat{\beta}_{j}(d) \triangleq\left[d^{H_{0}+H_{j}}\right]\left[\sum_{n} r_{0}(t, 1) r_{j}(t, 1)\right] / d^{2 H_{0}}\left[\sum_{n} r_{0}(t, 1)^{2}\right] \triangleq\left[d^{H_{0}+H_{j}}\right] / d^{2 H_{0}} \hat{\beta}_{j}
$$

donde los estimadores de la varianza y la covarianza están dados por (14) y (15):

$$
\begin{gathered}
\sum_{n} \frac{r_{0}(t, d)^{2}}{n} \triangleq d^{2 H_{0}}\left[\sum_{n} \frac{r_{0}(t, 1)^{2}}{n}\right] \\
\sum_{n} \frac{r_{0}(t, d) r_{j}(t, d)}{n} \triangleq d^{H_{0}+H_{j}}\left[\sum_{n} \frac{r_{0}(t, 1) r_{j}(t, 1)}{n}\right]
\end{gathered}
$$


Los estimadores mencionados anteriormente, muestran el efecto que generan los procesos de memoria de largo plazo sobre cada uno de los parámetros de segundo orden y, por consiguiente, sobre el parámetro beta, afectando el proceso de optimización de los portafolios, en términos de la estimación de la rentabilidad y el riesgo. Si se asume independencia $H=\frac{1}{2}$ para estimar cada uno de los parámetros mencionados, se obtiene el mismo resultado que ha desarrollado la teoría clásica de Markowitz con sus estimadores de covarianza, valor esperado, varianza de los retornos, así como del riesgo sistemático. La diferencia de la metodología propuesta frente a la tradicional se presenta en la inclusión del patrón de persistencia o memoria de largo plazo a los procesos generadores de las series de retornos, lo que claramente, como se ha expuesto, afecta las estimaciones de los parámetros.

\section{Estimación e inferencia del parámetro H.}

El parámetro de Hurst describe el comportamiento de persistencia en las observaciones de una serie en un intervalo de tiempo, conocido como memoria de largo plazo. Se puede estimar con ayuda del método del rango reescalado, desarrollado por Hurst en primera instancia, y luego trabajado por Mandelbrot (1972). Willinger et al. (1999) y también Grimm y Schluechtermann (2008), ofrecen más detalles acerca del método de estimación y de cómo este patrón de persistencia se incorpora y se asocia con los procesos ARIMA fraccionales.

Aunque existen varios métodos de estimación de $H$, el método del rango reescalado es uno de los más usados. Para un proceso $\left(X_{k}\right)$ de variables aleatorias, se calcula una variable $Y_{t}=\sum_{k=1}^{t} X_{k}$ para $t \in \mathbb{N}$, que representa la suma de carga del proceso en el tiempo $t$ teniendo en cuenta lo expuesto por Grimm y Schluechtermann (2008). A partir de lo anterior, se define el rango ajustado como en (16):

$$
R(l, k)=\max _{1 \leq j \leq k}\left(Y_{l+j}-Y_{l}-\frac{j}{k}\left(Y_{l+k}-Y_{l}\right)\right)-\min _{1 \leq j \leq k}\left(Y_{l+j}-Y_{l}-\frac{j}{k}\left(Y_{l+k}-Y_{l}\right)\right)
$$

con desviación estándar del proceso (17),

$$
S(l, k)=\left(\frac{1}{k} \sum_{j=l+1}^{l+k}\left(X_{j}-\hat{X}_{l, k}\right)^{2}\right)^{\frac{1}{2}}
$$

donde $\widehat{X}_{l, k}=\frac{1}{k} \sum_{j=l+1}^{l+k} X_{j}$ representa la media del $l$ ésimo bloque.

Con base en lo anterior se obtiene la fracción $Q(l, k)=\frac{R(l, k)}{S(l, k)}$ como rango reescalado.

$$
Q(l, k)=\frac{R(l, k)}{S(l, k)}
$$

Ahora, si se asume un proceso estocástico estacionario $\left(X_{k}\right)$ en estricto sentido, tal que $X_{k}^{2}$ es ergódico y $\frac{1}{n} \sum_{j=1}^{|n t|} X_{j}$ converge débilmente a un $\mathrm{fBm}$ con parámetro $H$, entonces para $k \rightarrow \infty$, $k^{H} Q(k) \sim \xi$, converge en distribución. Por lo que se puede establecer vía mínimos cuadrados ordinarios una estimación de $H$, como la pendiente del modelo $\log Q(k)$ en función de $\log k$, para un número adecuado de diferentes valores de $k$ y $l$.

\subsection{Rango reescalado y rango reescalado modificado: inferencia sobre $\boldsymbol{H}$.}

Lo (1991) complementa el estimador de la varianza muestral planteado por Mandelbrot (1972) incorporándole pesos a las covarianzas de los bloques generados. En su análisis, Lo (1991) afirma que, al establecer una hipótesis nula de independencia, se puede incrementar el error de tipo I, en la medida en que el estimador de la varianza sea sesgado al desconocer la autocorrelación como lo plantea 
Mandelbrot (1972) en su propuesta. Además, este estimador no puede distinguir entre corto y largo plazo, afectando los procesos de estimación y de inferencia.

De acuerdo con lo anterior, la ecuación que plantea Lo (1991) está dada por:

$$
\tilde{Q}(l, k)=\frac{1}{\widehat{\sigma}_{k}^{2}(q)} \max _{1 \leq j \leq k}\left(Y_{l+j}-Y_{l}-\frac{j}{k}\left(Y_{l+k}-Y_{l}\right)\right)-\min _{1 \leq j \leq k}\left(Y_{l+j}-Y_{l}-\frac{j}{k}\left(Y_{l+k}-Y_{l}\right)\right)
$$

En donde el estimador de varianza reconoce la autocorrelación como un patrón del proceso generador:

$$
\begin{gathered}
\hat{\sigma}_{k}^{2}(q) \equiv \frac{1}{k} \sum_{j=l+1}^{l+k}\left(X_{j}-\hat{X}_{l, k}\right)^{2}+\frac{2}{k} \sum_{j=1}^{q} \omega_{j}(q)\left\{\sum_{i=j+1}^{q}\left(X_{i}-\bar{X}_{k}\right)\left(X_{i-j}-\bar{X}_{k}\right)\right\} ; \omega_{j}(q)=1-\left|\frac{j}{q+1}\right| \\
q<k
\end{gathered}
$$

En términos inferenciales, para validar si el patrón de persistencia es significativo, siguiendo a Lo (1991) y Willinger et al. (1999) se utilizan intervalos de confianza. Al respecto, se requiere definir el rezago $q$ necesario para mejorar la estimación del rango reescalado, que se denomina rango reescalado modificado donde el $q$ óptimo se calcula de acuerdo con Teverovsky et al. (1999) como:

$$
q=\left[k_{n}\right], \quad k_{n} \equiv\left(\frac{3 n}{2}\right)^{\frac{1}{3}}\left(\frac{2 \widehat{\rho}}{1-\widehat{\rho}^{2}}\right)^{\frac{2}{3}}
$$

Como se plantea en Teverovsky et al. (1999) se sugiere que la serie de tiempo no presenta persistencia si el valor del estadístico se encuentra en el intervalo establecido por los límites $0.809 \mathrm{y}$ 1.862. El estadístico se presenta en (22):

$$
V_{q}(n)=n^{\frac{1}{2}} R(n) / \hat{\sigma}_{q}(n)
$$

tal que $\lim _{n \rightarrow \infty} P\left\{V_{q}(n) \in[0.809,1.862]\right\}=0.95$

Willinger et al. (1999) afirman que dicha prueba puede ser válida si para la estimación de $q$ se elaboran algunos gráficos complementarios con diferentes valores de $q$ para $V_{q}(n)$, que corroboren las conclusiones de la prueba. También, es posible realizar el ajuste de Anis y Lloyd (1976), derivado de una versión mejorada de Weron (2002), en donde $H$ se sugiere estimar como 0.5 más la pendiente de $(R / S)_{n}-E(R / S)_{n}$, donde,

$$
E(R / S)_{n}=\frac{n-\frac{1}{2}}{n} \frac{1}{\sqrt{n \frac{\pi}{2}}} \sum_{i=1}^{n-1} \sqrt{\frac{n-i}{i}}
$$

para $n>340$, que corresponde a nuestro caso. Sin embargo, dado que en ocasiones con este ajuste se puede llegar a estimaciones no esperadas superiores a uno, se modifica el ajuste de acuerdo con Sánchez, Trinidad y García (2008), quienes sugieren hallar la pendiente de la regresión lineal simple de $\log (R / S)_{n}-\log E(R / S)_{n}+\log (n) / 2$ sobre $\log (n)$.

Para complementar la inferencia sobre $H$ se usan también los intervalos de confianza empíricos de Anis y Lloyd (1976) sobre el R/S corregido a un nivel del 95\%, cuyos límites vienen dados por:

$$
0.5-\exp \left(-7.33 \log \left(\log \left(\log _{2} n^{*}\right)\right)+4.21\right) \text { y } 0.5+\exp \left(-7.20 \log \left(\log \left(\log _{2} n^{*}\right)\right)+4.04\right)
$$




\subsection{Método de estimación de Higuchi.}

Entre los métodos que procesan la varianza para evaluar la presencia de memoria de largo plazo se encuentra el de Higuchi, que fracciona las series en bloques siguiendo la presentación de Montanari, Taqqu y Teverovsky (1999). Estos sugieren que la serie toma la suma parcial de $Y(n)=\sum_{i=1}^{n} X_{i}$ de la serie original de donde:

$$
L(m)=\frac{n-1}{m^{3}} \sum_{i=1}^{m}\left[\frac{n-i}{m}\right]^{-1[(N-i) / m]} \mid Y(i+k m)-Y(i+(k-1) m \mid
$$

donde $n$ hace referencia al tamaño de la muestra, que se divide en bloques de tamaño $m$. El valor del parámetro $H$ se obtiene con ayuda de la regresión log-log de $L(m)$ sobre $m$, en donde el valor de la pendiente es igual a $D=2-H$.

\section{Estado del arte.}

En América Latina se han realizado varios estudios que buscan encontrar un patrón de persistencia de largo plazo de las series de retornos de algunas acciones e índices bursátiles. En Colombia, León y Vivas (2010) y León y Reveiz (2011), entre otros autores, muestran que la existencia de dependencia serial de largo plazo de los retornos viola el supuesto de independencia y utilizan la metodología del rango reescalado y el exponente de Hurst para analizar dicha dependencia en algunas series financieras.

León y Vivas (2010) estiman la presencia de memoria de largo plazo en los mercados cambiario, accionario y de renta fija colombianos; para ello, utilizan la metodología de rango reescalado clásico y modificado. Además de encontrar persistencia significativa para el mercado accionario y de renta fija, concluyen que el supuesto según el cual los precios recogen toda la información disponible es errado y que algunas técnicas empleadas en la optimización de portafolios y la valoración de activos son cuestionables.

Como parte de su investigación, Leiton (2011) analiza tres acciones de mayor bursatilidad en el mercado colombiano y el IGBC y a partir del cálculo de los exponentes de Hurst concluye sobre la existencia de persistencia en todas las series. Sin embargo, cuando realiza pruebas de significancia a los exponentes de Hurst estimados, encuentra que solo la acción de Bancolombia y el IGBC rechazan la hipótesis nula de independencia.

Duarte, Sierra y Mascareñas (2014) prueban la existencia de comportamiento caótico en las principales series bursátiles de Colombia usando el coeficiente de Hurst. Concluyen que las series bursátiles colombianas en su mayoría no son normales y presentan persistencia, resultado que apoya la hipótesis de existencia de un mercado fractal. Además, observan que el comportamiento de las series puede ser explicado tanto por modelos lineales ARIMA-GARCH como por modelos no lineales que se ajustan mejor al comportamiento caótico, esto como resultado de los coeficientes de Hurst obtenidos para la mayoría de los activos analizados.

De todas maneras, citando investigaciones anteriores hechas para Colombia, resaltan la obtención de resultados contradictorios:

es así como Ceretta \& Frois (2006) encuentran en este mercado, dependencia de largo plazo con correlación negativa (antipersistencia) en el periodo de 1994 a 2005; mientras Lim \& Brooks (2010), Restrepo \& Vásquez (2011) y Leiton (2011), hallan persistencia al evaluar el índice en los periodos de 1995 a 2005, 2001 a 2011 y 1998 a 2011, respectivamente; además Leiton (2011) también encuentra persistencia en las series de las acciones Nutresa, Éxito y Bancolombia durante el periodo estudiado. A pesar de estas contradicciones, todos los autores coinciden en que el mercado colombiano tiene memoria de largo plazo y presenta evidencia de multifractalidad (Zunino, Tabak, 
Pérez, Garavaglia, \& Rosso, 2007; Zuninoa et al., 2009) como indicio de comportamiento caótico, lo cual va en contra de la hipótesis de eficiencia del mercado planteada por Fama (1970). (Duarte, Sierra y Mascareñas, 2014, p. 3)

Una investigación posterior de Domínguez (2016) hecha para los precios de las cinco principales acciones del Mercado Bursátil colombiano entre 2011 y 2014 llega a las mismas conclusiones de Duarte et al. (2014); concluye sobre la presencia de multifractalidad tanto en las series como en sus correlaciones, sugiriendo el incumplimiento de la hipótesis de los mercados eficientes en favor de la existencia de un mercado fractal. Un trabajo similar de Nieto, Álvarez y Rodríguez (2016) fue realizado para cuatro acciones colombianas - periodo 2008-2012 - bajo la metodología de rango reescalado y exponente de Hurst. Concluyen que los activos analizados presentan fenómenos de persistencia y antipersistencia lo que desmiente la hipótesis de los mercados eficientes y que la trayectoria de los precios siga un comportamiento browniano.

Estudios hechos en otros países de América Latina confirman también la presencia de dependencia de largo plazo de los rendimientos de los activos financieros. Así lo demuestra el trabajo de López, Villagómez y Venegas (2011), quienes observaron el comportamiento de los valores diarios de cierre de las series de rendimientos del Índice de Precios y las cotizaciones de la Bolsa Mexicana de Valores desde enero de 1983 hasta el 29 de mayo de 2009. Para el efecto, estiman el coeficiente de Hurst y realizan la prueba de rango reescalado, de acuerdo con la modificación propuesta por Lo (1991), y concluyen que, para los activos analizados, existe un proceso de memoria larga, tanto en los rendimientos como en sus volatilidades, lo que confirma resultados de investigaciones previas realizadas por Islas y Venegas (2003), Venegas e Islas (2005) y López, Venegas y Sánchez (2009), citado por López et al. (2011). Al igual que investigaciones anteriores encuentran que las series de rendimientos muestran una estructura no lineal y sugieren esta modelación frente al formato lineal, que resulta poco convincente, por ejemplo, aquella que se especifica como un proceso tipo ARMA.

Un estudio similar fue elaborado por Caberra, López y López (2012), quienes realizan un análisis de los rendimientos de cinco acciones de la Bolsa Mexicana de Valores y un tracker referente a activos subyacentes de opciones que se negocian en dicho mercado. Ellos buscan evidencia de la presencia de dependencia de largo plazo en dichos rendimientos y en sus volatilidades, a partir del análisis del estadístico R/S o rango reescalado para estimar el parámetro H y de los estimadores de Geweke y PorterHudak. Las pruebas realizadas "sugieren que únicamente los rendimientos de algunos activos mexicanos podrían caracterizarse como procesos de memoria larga, en tanto que los rendimientos de otros activos podrían caracterizarse como procesos con reversión a la media" (Caberra et al. 2012, p. 74). Sin embargo, advierten que sus resultados no son concluyentes, ya que mientras las pruebas de Hurst sugieren dependencia de largo plazo de los rendimientos de la mayoría de los activos analizados, de acuerdo con las demás pruebas realizadas la evidencia de memoria larga es muy débil.

Acuña y Álvarez (2017) mediante un análisis realizado para el periodo 2002 - 2014 del Índice de Precios Selectivo de Acciones del mercado bursátil de Chile (IPSA), concluyen sobre la existencia de memoria de largo plazo, tanto en valores como en sus rendimientos, mediante el cálculo del coeficiente de Hurst y Hurst ajustado y del análisis de estructuras caóticas.

Otros trabajos recientes que, si bien no se relacionan directamente con la metodología de estudio, hacen referencia a la conformación de portafolios y sus conclusiones son importantes en términos de la relación riesgo-rendimiento. Por ejemplo, Quiroga y Villalobos (2016) mediante un análisis de componentes principales y análisis discriminante lineal, clasifican 88 empresas de la Bolsa Mexicana de Valores en tres grupos que comparten similitudes en cuanto a su comportamiento bursátil para el periodo enero de 2015 a marzo de 2016. Concluyen que cada grupo mantiene correlación con sus integrantes, pero no con los elementos de otros grupos, comportamiento que aporta para la conformación de portafolios con riesgo diversificado en donde sus componentes no mantengan correlaciones entre sí. 
Samaniego y Rodríguez (2018) evalúan el desempeño de cuatro de los principales índices bursátiles de México, con el fin de seleccionar el mejor para una estrategia de inversión pasiva. Tomando como referencia la razón de Sortino, proponen la sustitución del exceso de retorno promedio por una probabilidad de acierto y la máxima desviación estándar sobre el retorno objetivo negativo. Concluyen que, con los ajustes mencionados, se obtienen resultados diferentes al índice de Sortino, siendo el IPC large cap el mejor índice en términos de su relación riesgo-rendimiento.

Luna y Agudelo (2019) aplican el modelo de Black-Litterman para el mercado de renta variable del MILA y evalúan si un portafolio así constituido mejora el rendimiento promedio y el alfa frente a alternativas pasivas; toman información trimestral para el periodo enero de 2009 y diciembre de 2016. Concluyen que en todos los casos los portafolios conformados con Black-Litterman agregan valor, tanto en términos de rendimiento promedio como de alfa. Y cuando estructuran un portafolio Black-Litterman regional con los activos de cada país, se comprueba que éste mejora un Benchmark regional, que se construye como un portafolio que contiene los cuatro índices, ambos medidos en dólares.

A nivel internacional se encuentran trabajos como el de Cajueiro y Tabak (2007), aunque no directamente en renta variable, encuentran que las tasas de interés London Interbank offered rates (LIBOR), uno de los referentes mundiales, presenta una estructura de dependencia de largo plazo, sugiriendo incorporar este patrón, principalmente en portafolios de renta fija.

Cajueiro y Tabak (2008) a través de las estimaciones de Hurst mediante Rango reescalado (R/S) y varianza reescalada $(\mathrm{V} / \mathrm{S})$, muestran la necesidad de incluir el patrón de persistencia en los modelos de precios de Black-Scholes, en la optimización de portafolios y en la administración del riesgo. En la misma línea, trabajos como los de Kristoufek y Vosvrda (2013), proponen una nueva medida de eficiencia del mercado, incorporando la estructura de correlación de los retornos en el corto y largo plazo a través del exponente de Hurst. Lo anterior, aplicado a índices de portafolios de 41 mercados, encontrando a los latinoamericanos como los menos eficientes. Se infiere sobre la necesidad de incorporar el patrón de persistencia en la optimización de portafolios, en particular para portafolios del mercado MILA.

Analizando la eficiencia de los mercados, el trabajo de Sensoy (2013) también aporta evidencia a la necesidad de incorporar el patrón de dependencia de largo plazo; en su estudio de los 19 miembros de The Federation of Euro-Asian Stock Exchanges (FEAS), a través del análisis del exponente de Hurst generalizado con datos diarios, encuentra diferentes grados de persistencia de los mercados miembros. Por su parte, Srbek (2018) llega a una conclusión similar analizando 27 stock índices, también mediante el exponente de Hurst, calculado a través del rango reescalado.

Auer y Hoffman (2016) a través de la estimación del exponente de Hurst mediante el rango reescalado, examinan la estructura de dependencia de largo plazo de las divisas para mercados desarrollados y emergentes. Muestran que la constante de Hurst contiene información valiosa para los inversionistas, específicamente con las estrategias de carry trade.

Urquhart (2016) en el mercado de commodities, encuentra también, con algunas estadísticas que incluyen el exponente de Hurst, cómo los retornos de algunos metales preciosos pueden ser predecibles en una estructura de tiempo variante, aportando así evidencia a la hipótesis de mercados adaptativos.

Más recientemente, Guasoni, Nika y Rásonyi (2019) muestran teóricamente y con datos simulados, cómo sacar ventaja de incorporar una estructura fraccional en los precios mediante el exponente de Hurst, en un modelo de movimiento Browniano fraccional. 


\section{Datos y metodología.}

Para esta propuesta se tomaron activos que cotizan en el MILA (Mercado Integrado Latinoamericano) y el Standard and Poors (S\&P) con información extraída de Economática quienes proporcionan el histórico de precios y rendimientos de cada una de las acciones y los índices.

El trabajo empírico se realizó en dos partes. En la primera, se estimó la persistencia, a partir del parámetro de Hurst. Para esto se utilizó el rango reescalado y el rango reescalado modificado, siguiendo el método presentado en Pfaff (2008). Se realizó también la prueba de Anis y Lloyd (1976) usando el procedimiento de Weron (2002). Para la prueba de inferencia del $q$ óptimo de Lo (1991) se incorporaron los desarrollos y cuestionamientos propuestos por Teverovsky et al. (1999) y Willinger et al. (1999). También se realizó la estimación de $H$ a través del método de Higuchi. Todas estas aproximaciones se describieron en el apartado 4 de este trabajo.

En la segunda parte, se realizó el proceso de optimización con 510 observaciones de cada variable, entre enero de 2018 y mayo de 2020; como el objetivo es conformar un portafolio mixto, se diseñó un portafolio con 3 acciones por país, para un total de 12 .

A partir de los cálculos de $H$ y su inferencia, se estimaron los betas ajustados de cada activo reconociendo el patrón de persistencia de acuerdo con (13), (14) y (15). Se optimizaron los portafolios en función de los betas estimados como medida de riesgo sistemático, de acuerdo con (25). Los betas se estimaron tomando como referentes el MILA y el S\&P y su correspondiente estimación e inferencia del patrón $H$ de persistencia. Se utilizaron estos dos referentes de comportamiento de mercado.

La formulación matemática del problema de optimización estuvo dada por:

$$
\max \frac{\sum_{j} w_{j} \bar{R}_{j}(t, d)}{\beta_{p}} ; \beta_{p}=\sum_{j} w_{j} \widehat{\beta}_{J}(d), 0<w_{j}<1, \sum_{j} w_{j}=1
$$

En resumen, primero se hizo el proceso de inferencia sobre $H$ para cada uno de los activos analizados y luego se procedió a optimizar los portafolios a partir de estos resultados. Finalmente, el backtesting se hizo en una ventana histórica que permitió la calibración de cada estrategia de portafolios cada 22 días, para un total de 24 procesos de optimización, en dos años. Lo anterior, replicable en 4 estrategias para la inclusión del riesgo sistemático y el patrón de persistencia, MILA con y sin persistencia, y $\mathrm{S} \& \mathrm{P}$ con y sin persistencia. Se realizó una evaluación histórica de rentabilidades y se compararon las curvas de frontera eficiente (beta vs rentabilidad) para evaluar el desempeño de las 4 alternativas.

Cabe mencionar que la optimización de portafolios tuvo en cuenta el valor de $H$ para cada acción en particular, de acuerdo con las pruebas de inferencia que permitieron incluir el patrón de persistencia; el resto de las acciones incluyó el valor de $H$ asociado a independencia.

\section{Resultados.}

De acuerdo con la metodología mencionada, en la Tabla 1 se resume el proceso de estimación e inferencia desarrollados en el apartado 4. Se presentan por países los valores estimados del parámetro $H$, el resultado de la estimación del proceso Anis y Lloyd (1976)-HA, los intervalos de confianza para evaluar este parámetro, el $q$ óptimo, el valor de decaimiento $V q$ y, por último, la estimación a través del método de Higuchi. 
Tabla 1. Estimación del parámetro $H$ por el método del rango reescalado.

\begin{tabular}{|l|c|l|c|c|r|r|c|}
\hline VARIABLE & $\boldsymbol{H}$ & \multicolumn{1}{|c|}{$\boldsymbol{H} \boldsymbol{A}$} & $\boldsymbol{L} \boldsymbol{L}$ & $\boldsymbol{L S}$ & $\boldsymbol{q}$ Optimo & $\boldsymbol{V} \boldsymbol{q}$ & $\boldsymbol{H}$ Higuchi \\
\hline LUSUR & 0.6717 & $0.6715^{+++}$ & 0.4237 & 0.5726 & 4 & $4.176^{* * *}$ & 0.7532 \\
\hline CREDICORP & 0.6421 & $0.6420^{+++}$ & 0.4252 & 0.5712 & 3 & $3.383^{* * *}$ & 0.7163 \\
\hline VOLCAN & 0.6147 & $0.6146^{+++}$ & 0.4225 & 0.5737 & 7 & $2.348^{* * *}$ & 0.6437 \\
\hline RIPLEY & 0.5471 & 0.5467 & 0.4113 & 0.5842 & 5 & 1.362 & 0.4875 \\
\hline GASCO & 0.6662 & $0.6661^{+++}$ & 0.4272 & 0.5693 & 10 & $3.572^{* * *}$ & 0.6386 \\
\hline ITAUCORPBA & 0.6121 & $0.6119^{+++}$ & 0.4164 & 0.5794 & 4 & $2.623^{* * *}$ & 0.6833 \\
\hline ALFA & 0.6382 & $0.6381^{+++}$ & 0.4293 & 0.5674 & 8 & $3.374^{* * *}$ & 0.6791 \\
\hline ELEKTRA & 0.6522 & $0.6521^{+++}$ & 0.4268 & 0.5697 & 8 & $3.591^{* * *}$ & 0.6717 \\
\hline ICA & 0.5333 & 0.5330 & 0.4267 & 0.5698 & 7 & 1.379 & 0.4873 \\
\hline CEMARGOS & 0.6398 & $0.6397^{+++}$ & 0.4209 & 0.5752 & 6 & $3.266^{* * *}$ & 0.7169 \\
\hline ECOPETROL & 0.5626 & 0.5622 & 0.4046 & 0.5904 & 4 & 1.607 & 0.5492 \\
\hline EXITO & 0.6272 & $0.6270^{+++}$ & 0.4192 & 0.5768 & 1 & $2.935^{* * *}$ & 0.6749 \\
\hline MILA & 0.5919 & 0.5912 & 0.3512 & 0.6399 & 4 & 1.704 & 0.4615 \\
\hline S \&P & 0.6421 & $0.6421^{+++}$ & 0.4409 & 0.5565 & 3 & $3.917^{* * * *}$ & 0.7050 \\
\hline
\end{tabular}

${ }^{+++}$significativo para la prueba de Anis y Lloyd. ***significativo para la prueba de Lo ajustado por

Teverovsky et al. (1999).

Fuente: Elaboración propia.

En la primera columna se encuentran las 12 acciones en evaluación, 3 por país, en el siguiente orden: Perú, Chile, México y Colombia. En las últimas dos filas aparecen los dos índices de mercado que se usaron para el proceso de estimación: MILA y S\&P.

La segunda columna presenta los resultados del parámetro $H$ estimado usando el método que propone Pfaff (2008). Los valores de $H$ demuestran que algunas series financieras presentan persistencia estadística aunque con valores alrededor de $H=0.6$.

Las columnas 3, 4 y 5 resumen el ajuste de $H(H A)$ y la prueba de Anis y Lloyd (1976). Como ya se mencionó, el proceso de inferencia se hizo calculando los intervalos de confianza al $95 \%$ teniendo en cuenta la metodología utilizada en Weron (2002); si el valor de $H A$ cae dentro de ese intervalo se asume independencia de la serie; en caso contrario, se asume un proceso fraccional que puede ser persistente si cae por encima del intervalo, o anti persistente, si cae por debajo del intervalo.

El segundo proceso de inferencia de la Tabla 1 se encuentra en las columnas seis y siete y son los resultados de la prueba de Lo (1991) en donde se encuentra el $q$ óptimo y el valor inicial de decaimiento $V q$, ambos sirven como medida para evaluar la estimación del parámetro de Hurst con el método de rango reescalado de la columna número dos. Determinar el valor de $q$ y sobre todo el valor óptimo, es un proceso controvertido por la poca evidencia que prueba su eficacia como lo sugiere Pfaff (2008); por lo tanto, la propuesta de Teverovsky et al. (1999), fue la que se implementó para obtener este valor. En las publicaciones de Willinger et al. (1999) y de Teverovsky et al. (1999) se aprecia cómo encontrar el $q$ óptimo para analizar el efecto sobre el método de rango reescalado modificado que se aborda en la sección cuatro de este trabajo.

Con los resultados del $q$ Óptimo y el valor de decaimiento $V q$, es posible tomar la decisión de rechazar o no la hipótesis nula de independencia de la prueba de Lo (1991), ésta se evalúa teniendo en cuenta el punto en el que los valores de decaimiento de $V q$ cruzan uno de los dos límites de confianza propuestos en la prueba, que son 0.809 y 1.862 . En este trabajo se pudo constatar que, si la serie es 
persistente, el valor de decaimiento se encuentra por encima del límite superior,1.862. Si la serie se considera independiente, el valor de decaimiento cae dentro del intervalo.

De las catorce variables que se evaluaron en este trabajo (12 acciones y dos índices), solo cuatro presentaron un parámetro $H$ cercano a 0.5 , sugiriendo ausencia de persistencia; estas fueron: MILA, Ripley de Chile, ICA de México y Ecopetrol de Colombia. Las demás variables presentaron persistencia. Se resalta la congruencia de las dos pruebas utilizadas, llevando a similares conclusiones sobre la persistencia o no de las series de tiempo analizadas.

La última columna de la Tabla 1 contiene los resultados de la estimación del parámetro de Hurst a través del proceso alternativo de Higuchi; si bien los valores estimados del parámetro de Hurst por este método son diferentes a los presentados por el rango reescalado, estos coinciden en darle mayor fuerza a la persistencia estadística en las acciones que así lo demuestran.

A continuación, se analiza el efecto del parámetro de Hurst en los procesos de optimización de portafolios. Se comparan las curvas de frontera eficiente de los cuatro escenarios descritos en la metodología (Figura 1), donde se muestra su comportamiento promedio, que refleja la relación rentabilidad anual-riesgo para cada una de las estrategias planteadas, usando como referentes el mercado MILA y el S\&P bajo persistencia e independencia. Todos estos portafolios se construyeron a partir de los $H$ que se obtuvieron de la estimación por rango reescalado teniendo en cuenta los resultados inferenciales.

Figura 1. Análisis de rentabilidad anual - riesgo para diferentes portafolios.

Frontera Eficiente para Cuatro Escenarios

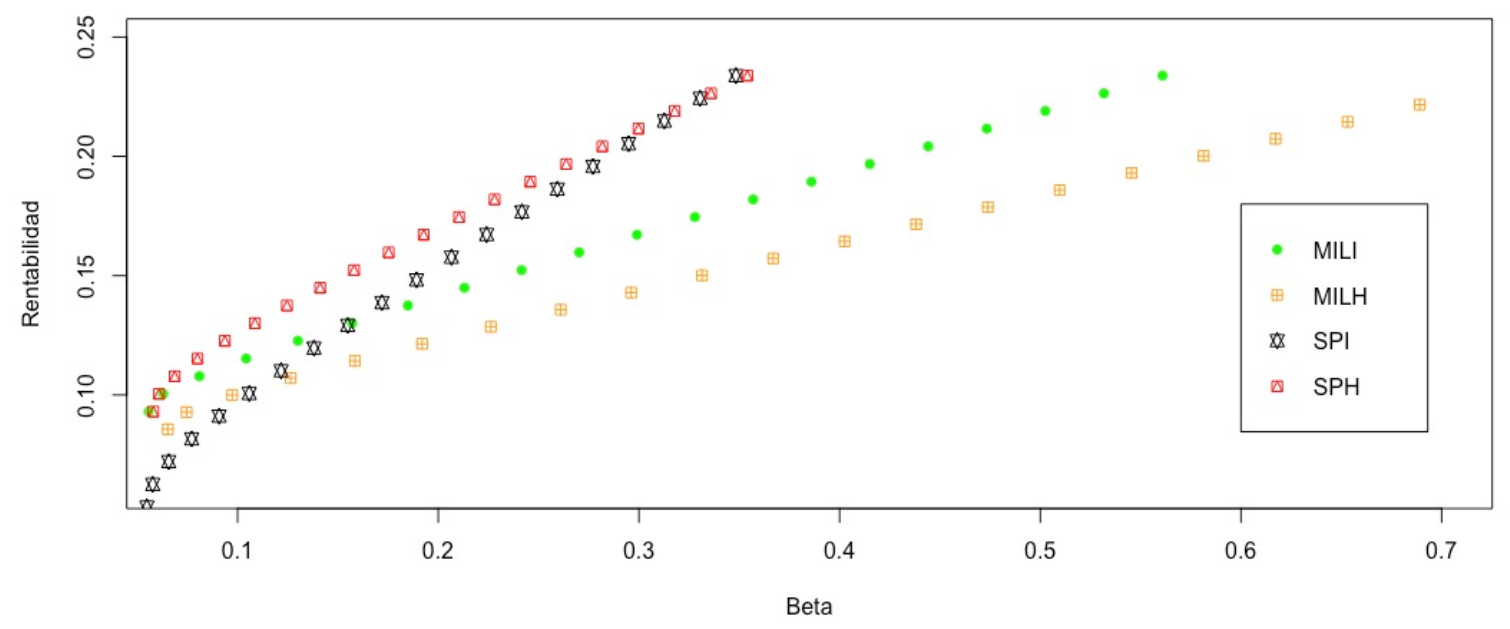

Fuente: Elaboración propia.

En el eje vertical de la Figura 1 se presenta la rentabilidad esperada anual, entre $0 \%$ y $25 \%$, mientras que en el eje horizontal se encuentra el coeficiente beta como medida de riesgo, entre 0 y 0.7 ; se destaca que el eje de los betas tiene valores inferiores a 1, hecho que coadyuva al proceso de minimización del riesgo. Para este período en particular, el mejor escenario correspondió al SPH (usando como referente el S\&P y teniendo en cuenta las pruebas inferenciales sobre $H$ ), quien presentó la mejor relación rentabilidad riesgo, seguido por el SPI (usando como referente el S\&P y asumiendo independencia), MILI (usando como referente el MILA y asumiendo independencia) y MILH (usando como referente el MILA y teniendo en cuenta las pruebas inferenciales sobre $H$ ). 
Se puede inferir que a medida que aumenta el riesgo, para el periodo en particular, se pierde el efecto de la persistencia en estos portafolios. Si el índice no presenta persistencia, como en este caso el MILA, es mejor evaluar los portafolios de forma tradicional, ya que, de otra manera, como se observa en la gráfica, las ponderaciones no van a mostrar los mejores resultados.

En general se observa que para el modelo de mercado es preferible usar como referente un mercado desarrollado como el S\&P y tener en cuenta la persistencia de las acciones cuando las pruebas así lo confirman, presentando resultados más eficientes, es decir, mayor rentabilidad por nivel de riesgo asumido.

Adicionalmente, como se mencionó en la metodología, para el proceso de backtesting se calcularon los rendimientos acumulados para cada escenario, en una ventana de dos años; los resultados se muestran en la Figura 2, con las mismas convenciones de la Figura 1. Se observa que dichos rendimientos en la mayor parte del periodo fueron superiores para el escenario SPH, ratificando lo encontrado a favor de usar como referente de mercado el S\&P, reconociendo el patrón de persistencia. Como hecho particular, es posible ver el efecto o impacto negativo que trajo el COVID 19 al comenzar el primer trimestre de 2020 en los mercados financieros latinoamericanos.

Figura 2. Backtesting.

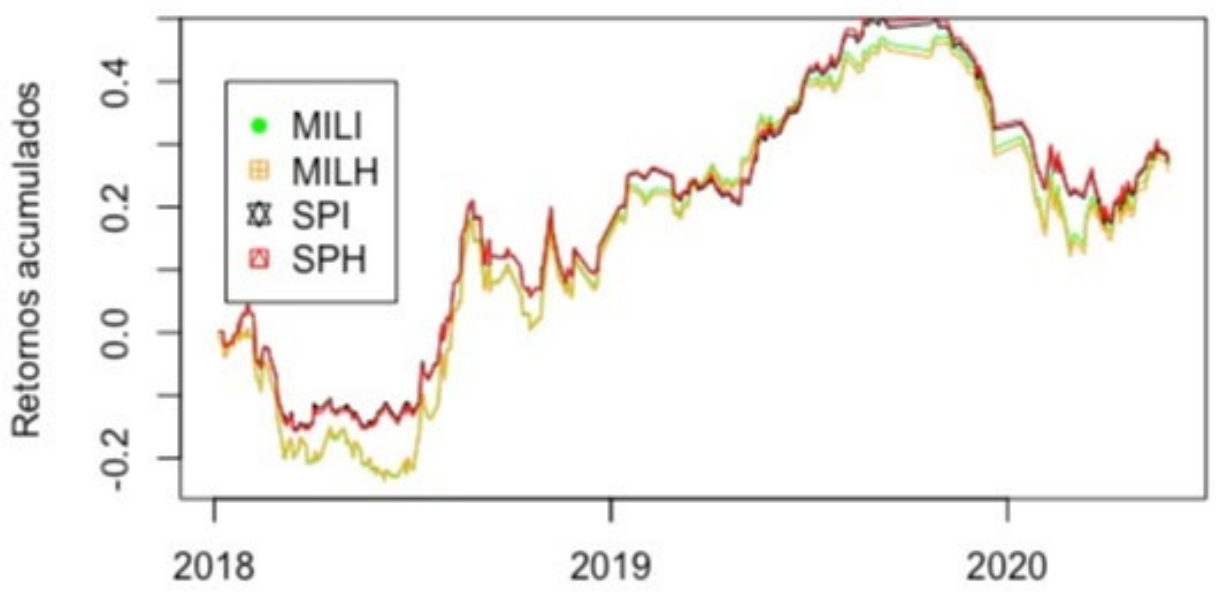

Fuente: Elaboración propia.

\section{Conclusiones.}

En general, las dos pruebas inferenciales utilizadas permiten llegar a conclusiones similares con respecto al patrón de persistencia de las series analizadas. En particular, el índice MILA tuvo una estimación de $H=0.59$, y la inferencia confirma que a pesar de que el valor es cercano a 0.6 , no existe evidencia de persistencia en las observaciones. Por el contrario, el S\&P y la mayoría de las acciones analizadas presentan dependencia, con excepción de Ripley de Chile, ICA de México y Ecopetrol de Colombia, que evidencian independencia.

Por supuesto, como lo sugirieron Willinger et al. (1999) y como se hizo en este trabajo, es muy importante realizar siempre una inferencia cuidadosa de $H$ que se ajuste a los patrones temporales de las series para una mejor estimación de $H$; esto se traduce en una mejor gestión del riesgo, lo que genera resultados más robustos derivados de la optimización de portafolios. 
Las estimaciones por el método de Higuchi también le dan fuerza a los hallazgos de persistencia de las series que estadísticamente muestran este patrón. Aunque se esperaba un comportamiento similar del índice MILA frente a las acciones que pertenecen a su mercado, éste no refleja qué sucede con las acciones que lo conforman, aspecto que puede ser objeto de discusión en otra investigación.

Los resultados del backtesting también comprueban que, al incluir el patrón de persistencia, solo cuando la inferencia así lo indica, genera mejores resultados. Los portafolios que presentan los mejores rendimientos a un menor riesgo son aquellos que toman como referente el S\&P, cuando se reconocen los patrones de persistencia; por el contrario, si se toma como referente el MILA, se obtienen mejores resultados bajo independencia, ya que éste mostró específicamente un claro patrón de no persistencia. En conclusión, se evidencia la necesidad de incorporar la persistencia en los procesos de optimización solo cuando las series presentan este patrón, lo que genera mejores resultados de desempeño en función de la relación riesgo-rendimiento.

Como trabajo posterior, se propone realizar bajo distintos escenarios un backtesting con una ventana de tiempo mayor para comprobar la eficiencia de cada uno de los portafolios en términos del patrón de persistencia, los que, al presentar fuertes cambios en la volatilidad de los rendimientos, evidencian la necesidad de incorporar este patrón.

\section{Referencias}

Acuña, C., \& Álvarez, A. (2017). Dependencia serial de largo plazo en el índice bursátil chileno, a través del coeficiente de Hurst y Hurst ajustado. Journal of Economics, Finance and Administrative Science, 22(42), 37-50.

Anis, A.A., \& Lloyd, E.H. (1976). The expected value of the adjusted rescaled Hurst range of independent normal summands. Biometrika, 63(1), 111-116.

Auer, B.R., \& Hoffmann, A. (2016). Do carry trade returns show signs of long memory? The Quarterly Review of Economics and Finance, 61, 201-208. https://doi.org/10.1016/j.qref.2016.02.007

Benth, F. (2003). On arbitrage-free pricing of weather derivatives based on fractional brownian motion. Applied Mathematical Finance, 10(4), 303-324.

Biagini, F., Campanino, M., \& Fuschini, S. (2008). Discrete approximation of stochastic integrals with respect to fractional Brownian motion of Hurst index $\mathrm{H}>1 / 2$. Stochastics An International Journal of Probability and Stochastic Processes, 80(5), 407-426.

Caberra, A.I., López, S.S., \& López, F. (2012). Dependencia de largo plazo en los rendimientos de acciones mexicanas selectas. Panorama Económico, 7(14), 59-78.

Cajueiro, D.O., \& Tabak, B.M. (2007). Long-range dependence and multifractality in the term structure of LIBOR interest rates. Physica A: Statistical Mechanics and Its Applications, 373, 603614. https://doi.org/10.1016/j.physa.2006.04.110

Cajueiro, D.O., \& Tabak, B.M. (2008). Testing for long-range dependence in world stock markets. Chaos, Solitons \& Fractals, 37(3), 918-927. https://doi.org/10.1016/j.chaos.2006.09.090

Cheridito, P. (2001). Mixed fractional Brownian motion. Bernoulli, 7(6), 913-934. https://projecteuclid.org/download/pdf_1/euclid.bj/1078951129 
Domínguez, A. (2016). Análisis multifractal de correlaciones en series temporales de sistemas económicos. Memoria del Trabajo de Fin de Máster, Universitat de Les Illes Balears, Palma de Mallorca.

Duarte, J., Sierra, K., \& Mascareñas, J.M. (2014). Evaluación de la Memoria de Largo Plazo del Mercado Bursátil Colombiano mediante el Coeficiente de Hurst (Evaluation of Long-Term Memory in Colombian Stock Market by Hurst Coefficient). Revista Internacional Administración \& Finanzas, 7(4), 1-10.

Greene, M., \& Fielitz, B. (1980). Long-term dependence and least squares regression in investment analysis. Management Science, 26(10), 1031-1038.

Grimm, C., \& Schluechtermann, G. (2008). IP-traffic theory and performance. Heidelberg: Springer.

Guasoni, P., Nika, Z., \& Rásonyi, M. (2019). Trading Fractional Brownian Motion. SIAM Journal on Financial Mathematics, 10(3), 769-789. https://doi.org/10.1137/17m113592x

Hu, Y., \& Øksendal, B. (2003). Fractional white noise calculus and applications to finance. Infinite Dimensional Analysis, Quantum Probability and Related Topics, 6(1), 1-32.

Kristoufek, L., \& Vosvrda, M. (2013). Measuring capital market efficiency: Global and local correlations structure. Physica A: Statistical Mechanics and Its Applications, 392(1), 184193. https://doi.org/10.1016/j.physa.2012.08.003

Leiton, K. (2011). Validez del supuesto de neutralidad del horizonte de tiempo en el CAPM y la metodología del rango reescalado: aplicación a Colombia. Borradores de Economía, 672, 1-42. https://doi.org/10.32468/be.672

León, C., \& Reveiz, A. (2011). Portfolio Optimization and Long-Term Dependence. Banco de la Republica Working Paper, 602, 1-26. http://dx.doi.org/10.2139/ssrn.1686115

León, C., \& Vivas, F. (2010). Dependencia de largo plazo y la regla de la raiz del tiempo para escalar la volatilidad en el mercado colombiano. Borradores de Economía, 603, 1-47. https://doi.org/10.32468/be.603

Lo, A. (1991). Long-term Memory in Stock Market Prices. Econometrica, 59 (5), 1279-1313.

López, F., Villagómez, J., \& Venegas, F. (2011). Evidencias de memoria larga en el Índice de Precios y. En M. d. Martínez, C. Zubieta, \& F. López, Administración del Riesgo (2 ed.). México: Azcapotzalco.

Luna, S., \& Agudelo. D. (2019). ¿Agrega Valor el Modelo Black-Litterman en Portafolios del Mercado Integrado Latinoamericano (MILA)? Evaluación Empírica 2008-2016. Revista de Métodos Cuantitativos para la Economía y la Empresa, 27, 55-73.

Mandelbrot, B. (1972). Statistical methodology for nonperiodic cycles: from the covariance to $\mathrm{r} / \mathrm{s}$ analysis. Annals of Economic and Social Measurement, 1(3), 259-290.

Mandelbrot, B., \& Hudson, R. (2005). Fraktale und Finanzen: Märkte zwischen Risiko, Rendite und Ruin. München und Zürich: Piper.

Mandelbrot, B., \& Vann Ness, J. (1968). Fractional Brownian Motions, Fractional Noises and Applications. SIAM Review, 10(4), 422-437. 
Mandelbrot, B., \& Wallis, J. (1969). Computer Experiments with Fractional Noises. Water Resources Research, 5, 228-267.

Montarani, A., Taqqu, M \& Teverovsky, V. (1999). Estimating long-range dependence in the presence of periodicity: an empirical study. Mathematical Computer Modelling, 29(10-12), 2217-2228.

Nieto, H.D., Álvarez, J.E., \& Rodríguez, E.L. (2016). Análisis de persistencia en acciones financieras en el mercado colombiano a través de la metodología de Rango Reescalado (R/S). Cuadernos Latinoamericanos de Administración, 12(22), 23-32.

Nualart, D. (2003). Stochastic integration with respect to fractional brownian motion and applications. Contemporary Mathematics, 3-40. https://www.researchgate.net/profile/David_Nualart2/publication/242388658_Stochastic_integrati on_with_respect_to_the_fractional_Brownian_motion/links/542588c40cf238c6ea7414f0/Stochasti c-integration-with-respect-to-the-fractional-Brownian-motion.pdf

Pfaff, B. (2008). Analysis of integrated and cointegrated time series with R. New York: Springer Science \& Business Media.

Quiroga, C., \& Villalobos, A. (2016). Aplicación de dos técnicas del análisis multivariado en el mercado de valores mexicano. Revista de Métodos Cuantitativos para la Economía y la Empresa, 22, 104119.

Samaniego, Á., \& Rodríguez, L. (2018). Passive Portfolio Management by Indexing: A Performance Analysis of High, Medium and Low Capitalization Indices in Mexico. Revista de métodos cuantitativos para la economía y la empresa, 26, 269-293.

Sánchez, M.A., Trinidad, J.E., \& García, J. (2008). Some comments on Hurst exponent and the long memory processes on capital markets. Physica A: Statistical Mechanics and Its Applications, 387(22), 5543-5551. https://doi.org/10.1016/j.physa.2008.05.053

Sensoy, A. (2013). Time-varying long range dependence in market returns of FEAS members. Chaos, Solitons \& Fractals, 53, 39-45. https://doi.org/10.1016/j.chaos.2013.05.004

Sharpe, W. (1963). A Simplified Model for Portfolio Analysis. Management Science, 9(2), 277-293.

Srbek, P. (2018). Estimation of the Hurst Exponent in Time Series of Daily Returns of Stock Indices. Politicka Ekonomie, 66(4), 508-524.

Teverovsky, V., Taqqu, M.S., \& Willinger, W. (1999). A critical look at Lo's modified R/S statistic. Journal of statistical Planning and Inference, 80(1-2), 211-227.

Urquhart, A. (2016). How predictable are precious metal returns? The European Journal of Finance, 23(14), 1390-1413. https://doi.org/10.1080/1351847x.2016.1204334

Weron, R. (2002) Estimating long-range dependence: finite sample properties and confidence intervals. Physica A, 312, 285-299.

Willinger, W., Taqqu, M., \& Teverovsky, V. (1999). Stock market prices and long-range dependence. Finance and Stochastics, 3(1), 1-13. 